\title{
切向极化压电陶瓷晶堆中扭转振动的 传播速度研究
}

\author{
林书玉 \\ (陕西师范大学应用声学研究所, 西安 710062)
}

\section{关键词切向极化 扭转振动 截面扭转形状系数 传播速度}

随着超声马达、超声塑料焊接、超声疲劳试验以及超声旋转加工等新技术的发展, 扭转振 动超声换能器的理论及实验研究受到了普遍重视. 在功率超声领域, 类似于纵向振动夹心式 换能器的扭转振动夹心换能器具有结构简单、机电转换效率高等优点. 在纵向振动夹心式换 能器中, 压电激励元件为轴向极化的压电陶瓷晶堆, 而对于扭转振动夹心式换能器, 其激励元 件为切向极化的压电陶瓷晶堆. 文献 [1] 对夹心式扭转振动超声换能器进行了研究, 推出了 压电晶堆扭转振动的等效电路以及换能器的共振频率方程. 在此基础上, 本文对晶堆中的扭 转振动传播速度进行了进一步的研究, 试图为夹心式扭转振动超声换能器的理论及实验研究 提供较系统的设计理论.

\section{1 压电晶堆中扭转振动的传播速度研究}

根据文献 [1] 中的分析, 扭转振动在压电陶瓷晶堆中的传播速度为

$$
C_{e}=C_{t} \cdot\left[1-\left(K_{15}^{l}\right)^{2} \frac{\operatorname{tg} \frac{k_{t} l}{2}}{\frac{k_{t} l}{2}}\right]^{1 / 2},
$$

式中 $C_{\mathrm{t}}=\left[1 /\left(S_{55}^{D} \rho\right)\right]^{1 / 2}$ 为扭转振动在压电陶瓷细长管中的传播 速度, $k_{\mathrm{t}}=\omega / C_{\mathrm{t}}$ 为扭转振动的 波数, $l$ 为陶瓷晶堆中每一片压电元件的厚度, $K_{15}^{l}$ 为压电陶瓷细长棒中扭转振动的机电耦合 系数, 其表达式为

$$
K_{15}^{l}=\frac{g_{15} W}{\left.S_{55}^{E} \beta_{11}^{T} S I_{p}\right)^{1 / 2}}
$$

式中 $W=\iint_{s} r \mathrm{~d} s, I_{p}=\iint_{s} r^{2} \mathrm{~d} s, S=\pi\left(R^{2}-r^{2}\right)$ 为陶瓷晶堆的截面积, $R$ 和 $r$ 为圆环的内、外半 径. 由 (1) 式可以看出, 当晶堆中单个元件的厚度 $l$ 远小于扭转振动的波长 $\lambda$ 时, 即 $l \ll \lambda$, 可 得 $k_{t} l=\frac{2 \pi l}{\lambda} \ll 1, \operatorname{tg} \frac{k_{t} l}{2} \approx \frac{k_{t} l}{2}$, 因此(1)式可简化为

1995-04-04 收稿, 1995-08-03 收修改稿 


$$
C_{e}=C_{t}\left[1-\left(K_{15}^{1}\right)^{2}\right]^{1 / 2} \text {. }
$$

令 $\tau=\left(S I_{p} / W^{2}\right)^{1 / 2}$, 称为扭转振动体的截面扭转形状系数, 由 $W$ 及 $I_{p}$ 的定义式可得圆环形截面 的截面扭转形状系数为

$$
\tau=\left[\frac{9\left(R^{2}+r^{2}\right)(R+r)^{2}}{8\left(R^{2}+R r+r^{2}\right)^{2}}\right]^{1 / 2} .
$$

令 $B=r / R$ 为圆环的内、外半径比, (4) 式可化为

$$
\tau=\left[\frac{9\left(1+B^{2}\right)(1+B)^{2}}{8\left(1+B+B^{2}\right)^{2}}\right]^{1 / 2} .
$$

对于实心圆柱, $B=0$, 由 (5) 式可得

$$
\tau=(9 / 8)^{1 / 2} .
$$

对于薄壁圆环, 内、外半径比较接近, $B=1$, 代人 (5) 式可得

$$
\tau=1 \text {. }
$$

对于其他情况, 圆环的形状介于实心圆柱和薄壁圆环之间, 由此可得出扭转振动体的截面扭 转形状系数的取值范围为

$$
1 \leqslant \tau \leqslant(9 / 8)^{1 / 2} .
$$

由截面扭转形状系数的定义式及上述分析可见, $\tau$ 仅与振动体的截面形状有关, 而与振子的材 料及其他参数无关. 把 $\tau$ 的定义式代人 (2) 式可得

$$
K_{15}^{l}=K_{15} / \tau \text {, }
$$

式中 $K_{15}=g_{15} /\left(S_{55}^{E} \beta_{11}^{T}\right)^{1 / 2}$ 为压电陶瓷矩形薄板的厚度剪切机电耦合系数. 由 (9)式可见, 压电陶 瓷细长管扭转振动的机电耦合系数与振动体的截面形状有关. 利用 (9) 式, 压电陶瓷晶堆中 扭转振动的传播速度可表示为下述形式:

$$
C_{e}=C_{t}\left(1-\frac{K_{15}^{2}}{\tau^{2}}\right)^{1 / 2}
$$

把(5)式代人(10)式可得

$$
C_{e}=C_{t}\left[1-K_{15}^{2} \cdot \frac{8\left(1+B+B^{2}\right)^{2}}{9\left(1+B^{2}\right)(1+B)^{2}}\right]^{1 / 2} .
$$

利用 (8) 式, 可以得出压电陶瓷晶堆中扭转振动传播速度的两个极限值. 当陶瓷截面为薄壁 圆环时, 其截面的扭转形状系数等于 1 , 由此可得陶瓷晶堆中扭转振动传播速度的最小值为

$$
C_{e \min }=C_{t}\left(1-K_{15}^{2}\right)^{1 / 2} \text {. }
$$

把 $C_{t}$ 及 $K_{15}$ 的表达式代人上式可得

$$
C_{e \min }=\left[1 /\left(S_{55}^{E} \rho\right)\right]^{1 / 2} .
$$

对于实心截面压电陶瓷晶堆, 其截面的扭转形状系数取上限, 即 $\tau=(9 / 8)^{1 / 2}$, 代人 (10)及 $(11)$ 式可 
得陶瓷晶堆中扭转振动传播速度的最大值为

$$
C_{e \max }=C_{t}\left(1-\frac{8}{9} K_{15}^{2}\right)^{1 / 2} .
$$

利用 (12) 及 (14) 二式可得, 晶堆中扭转振动的最大速度与最小速度之差值为

$$
C_{e \max }-C_{e \min }=C_{t}\left(\sqrt{1-\frac{8}{9}} K_{15}^{2}-\sqrt{1-K_{15}^{2}}\right) .
$$

由 (15) 式可见, 对于由不同的压电陶瓷材料组成的晶堆, 最大扭转振动速度与最小扭转振动 速度的差值是不同的, 材料的机电耦合系数越大, 这一差值越大, 机电耦合系数越小, 差值越 小. 对于不具有压电性能的各向同性材料, $K_{15}=0$, 这一差值等于零, 即各向同性材料细长棒 中的扭转振动传播速度是一个常数, 与振子的截面形状无关.

由上述分析可以看出, 压电陶瓷晶堆中扭转振动的传播速度不仅与材料参数有关, 而且与 振子的截面形状有关,这一点与晶堆中纵向振动的传播规律是完全不同的, 对于轴向极化的 压电陶瓷晶堆, 纵向振动的传播速度为 ${ }^{[2]}$

$$
C_{e l}=C_{D}\left(1-K_{33}^{2}\right)^{1 / 2},
$$

式中 $C_{D}=\left[1 /\left(\rho S_{33}^{D}\right)\right]^{1 / 2}$, 是纵向振动在轴向极化压电陶瓷细长棒中的传播速度, $K_{33}$ 为纵向机电 耦合系数, 利用 $K_{33}$ 的定义式, 即 $1-K_{33}^{2}=S_{33}^{D} / S_{33}^{E},(16)$ 式可化为

$$
C_{e l}=\left[1 /\left(\rho S_{33}^{E}\right)\right]^{1 / 2} .
$$

(17) 式就是用于决定轴向极化晶堆中纵向振动传播速度的数学表达式, 很显然, 这一速度仅 决定于振子的材料参数, 与振子的截面形状无关.

\section{2 实验}

为了从实验上研究本文所得理论的正确性, 本文进行了一些实验, 由于切向极化压电陶 瓷圆环的制作工艺比较复杂, 因此, 切向极化压电陶瓷圆环的制作采用以下方法, 即利用沿宽 度方向极化的矩形压电陶瓷薄长条, 通过切割及研磨后, 拼凑成近似为切向极化的压电陶瓷 圆环. 本文采用的压电陶瓷矩形薄长条的厚度为 $2.5 \mathrm{~mm}$, 因此压电陶瓷圆环的厚度也为 $2.5 \mathrm{~mm}$, 圆环的内、外半径有 3 种规格, 即 $\phi 30 \times \phi 24(\mathrm{~mm}), \phi 30 \times \phi 18(\mathrm{~mm})$ 以及 $\phi 30 \times \phi 12(\mathrm{~mm})$, 由于制作工艺的限制, 上述圆环并不是理想的圆环. 而本文中理论要求压电晶堆的总长度远 大于其横向尺寸, 因此, 将压电圆环制作成夹心式扭转振动换能器, 根据夹心式压电换能器的 纵向振动理论, 只要换能器的总体纵向尺寸远大于其横向尺寸, 而且换能器中每一片陶瓷圆 环的厚度远小于其波长, 便可以实现本文理论所需要的条件, 通过对夹心式扭转振动压电换 能器的共振频率进行测试, 就可以间接测量压电陶瓷晶堆中扭转振动的传播速度, 从而对本文中 理论进行验证. 为了简化计算及分析, 夹心式换能器设计成对称结构, 陶瓷片位于换能器中 间位置, 前后金属块的材料为超硬铝 (标准代号为 LC4), 其材料参数取为: $\rho=2700 \mathrm{~kg} / \mathrm{m}^{3}$, $G=2.67 \times 10^{10} \mathrm{~N} / \mathrm{m}^{2}$. 压电陶瓷材料为 PZT -4 , 其标准的材料参数为: $\rho=7500 \mathrm{~kg} / \mathrm{m}^{3}, S_{55}^{E}=39.0$ $\times 10^{-12} \mathrm{~m}^{2} / \mathrm{N}, K_{15}=0.71$, 按照夹心式压电换能器的频率设计公式及文中理论, 设计了 3 个夹心 式扭转换能器, 每个换能器含有两片陶瓷环, 其具体尺寸列于表 1 , 其中 $r$ 及 $R$ 为陶瓷环的内、 
表 1 夹心式扭转换能器的尺寸及频率测试结果

\begin{tabular}{ccccccccc}
\hline 换能器 & $R / \mathrm{mm}$ & $r / \mathrm{mm}$ & $l_{0} / \mathrm{mm}$ & $l / \mathrm{mm}$ & $C_{\boldsymbol{c}} / \mathrm{m} \cdot \mathrm{s}^{-1}$ & $f / \mathrm{Hz}$ & $f_{m} / \mathrm{Hz}$ & $\Delta / \%$ \\
\hline 1 & 15 & 12 & 2.5 & 50 & 1853 & 14462 & 13757 & 5.12 \\
2 & 15 & 9 & 2.5 & 50 & 1867 & 14856 & 13951 & 6.49 \\
3 & 15 & 6 & 2.5 & 50 & 1894 & 14964 & 13988 & 6.98 \\
\hline
\end{tabular}

外半径, $l_{0}$ 为每片陶瓷环的厚度, $C_{e}$ 为陶瓷堆中的扭转声速, $l$ 为金属盖板的长度, 金属盖板的 半径等于陶瓷环的外半径. 利用传输线法测量了换能器的共振频率, 结果见表 1 , 其中 $f$ 为理 论设计频率, $f_{m}$ 为实测频率, $\Delta=\left|f_{m}-f\right| / f_{m}$, 换能器为半波振子, 其节点位于换能器的几何中 心, 其频率设计公式为

$$
\operatorname{tg} k_{0} l_{0} \cdot \operatorname{tg} k l=\frac{Z_{0}}{Z}
$$

式中 $k_{0}=\omega / C_{e}, k=\omega / c, c=(G / \rho)^{1 / 2}, Z_{0}=\rho_{0} C_{e} I_{p 0}, Z=\rho C I_{p}, I_{p 0}=\frac{\pi}{2}\left(R^{4}-r^{4}\right), I_{p}=\frac{\pi R^{4}}{2}$. 为了 验证压电陶瓷晶堆的几何形状对其扭转振动速度的影响, 3 个换能器各部分的几何尺寸基本 相同, 不同之处在于陶瓷片的内半径 $r$, 表中 $C_{e}$ 的计算公式为文中的 (10) 式. 由表中数据可 以看出, 由于陶瓷圆环的内径不同, 导致陶瓷堆中声速不同, 从而使同一几何尺寸 (轴向长 度) 的换能器具有不同的共振频率. 另外, 从表中实验数据也可以看出, 换能器的测试频率与 计算频率基本符合, 并且换能器的频率随着陶瓷内径的变化规律与理论预测规律也是基本一 致的. 关于换能器频率测量的误差来源, 主要有以下几点: (1) 材料的标准值与实际值有差 异; (2) 压电陶瓷圆环的极化方向并不是沿着理想的圆周切线方向; (3) 换能器各元件之间 的粘接工艺影响频率 (为了避免预应力螺栓引起的频率误差, 换能器各元件之间是利用 502 速 粘胶粘连在一起的, 因为换能器测量时处于小信号状态, 故不会出现裂开现象).

从上述分析及实验可以看出, 陶瓷环的几何尺寸确实影响其扭转振动传播速度, 因而影响 换能器的共振频率. 然而这种几何尺寸引起的声速及频率的变化是很小的, 因此, 在换能器 的工程设计及实际计算时, 可把扭转振动在压电陶瓷晶堆中的传播速度 $C_{e}$ 近似用下式表 示:

$$
C_{e}=C_{t}\left(1-K_{15}^{2}\right)^{1 / 2}=\left[1 /\left(S_{55}^{E} \rho\right)\right]^{1 / 2} .
$$

(19) 式也可以看成是令截面扭转形状系数 $\tau=1$, 由(10)式而得出的, 根据 (8) 式, 由于 $\tau$ 接近于 1 , 因 此这一近似是允许的. 从这可以看出, 晶堆中扭转振动与纵向振动的传播速度表达式基本相 同, 不同之处在于: 对于扭转振动, 应把 $K_{33}$ 及 $S_{33}^{E}$ 换成 $K_{15}$ 及 $S_{55}^{E}$.

\section{3 结论}

本文研究了切向极化压电陶瓷晶堆中扭转振动的传播规律, 总结全文内容可得以下结 论:

(1) 得出了切向极化压电陶瓷晶堆中扭转振动传播速度的数学表达式.

(2)引进了振子的截面扭转形状系数的概念, 并得出了其取值范围, 该系数仅与振子的截 面形状有关. 
(3) 晶堆中扭转振动的传播速度不仅决定于振子的材料系数, 而且与振子的截面形状有 关.

(4) 得出了晶堆中扭转振动速度的最大值与最小值, 并给出了二者之差.

(5) 理论要求晶堆的尺寸满足以下条件, 即晶堆的横向尺寸远小于其纵向尺寸以及扭转波 波长, 而晶堆中每一片元件的厚度也应远小于扭转波波长.

(6) 尽管晶堆中扭转振动的传播速度依赖于振子的几何形状及尺寸, 但由于这一影响很 小, 因此, 在扭转换能器的实际设计及计算时, 可以忽略形状及尺寸对振速的影响.

\section{参考 文 献}

1 Lin Shuyu. Study of the sandwiched piezoelectric ultrasonic torsional transducer. Ultrasonics, 1994, 32(6): $461 \sim 469$

2 Martin G E. On the theory of segmented electromechanical systems. J Acoust Soc Am, 1964, 36(7): $1366 \sim 1370$ 